\title{
ON INTERSECTION OF COMPACTA IN EUCLIDEAN SPACE II
}

\author{
A. N. DRANISHNIKOV
}

(Communicated by James E. West)

\begin{abstract}
Suppose that $X$ is a compact subset of $n$-dimensional Euclidean space $\mathbb{R}^{n}$. If every map $f: Y \rightarrow \mathbb{R}^{n}$ of a compactum $Y$ can be approximated by a map avoiding $X$ then $\operatorname{dim} X \times Y<n$.
\end{abstract}

\section{INTRODUCTION}

The main result of this paper is the following Theorem 1 which is the inverse of the main result of [D1].

Theorem 1. Let $X$ be a compactum in Euclidean space $\mathbb{R}^{n}$ and suppose that for a compact metric space $Y$, the space of maps $C\left(Y, \mathbb{R}^{n}-X\right)$ is dense in $C\left(Y, \mathbb{R}^{n}\right)$. Then $\operatorname{dim} X \times Y<n$.

Here $C(X, Z)$ denotes the space of continuous maps between $X$ and $Z$ with the compact-open topology.

This theorem plus Theorem 1 from [D1] implies the following:

Theorem 2. For an arbitrary compactum $Y$ and for a codimension three tame compact subset $X \subset \mathbb{R}^{n}$ the following are equivalent:

(a) $C\left(Y, \mathbb{R}^{n}-X\right)$ is dense in $C\left(Y, \mathbb{R}^{n}\right)$,

(b) $\operatorname{dim} X \times Y<n$.

Remark 1 [D2]. There exist compacta $X, Y$ with $\operatorname{dim} X=\operatorname{dim} Y=n-2$ and $\operatorname{dim} X \times Y<n$ for arbitrary $n$.

The proof of Theorem 1 is based on Spanier-Whitehead duality and some elements of extension theory. The top of the preliminary work in extension

Received by the editors April 23, 1990.

1980 Mathematics Subject Classification (1985 Revision). Primary 54C25, 55M10.

Key words and phrases. Compactum, cohomological dimension, Spanier-Whitehead duality, unstable intersection.

The author was partially supported by the U.S. Army Research Office through the Mathematical Sciences Institute of Cornell University. 
theory is Theorem 3 which is a generalization of the inequality $\operatorname{dim} \geq \operatorname{dim}_{S}$, where $\operatorname{dim}_{S}$ is the stable cohomotopy dimension [D3].

\section{EXTENSION THEORY}

Notation [Ku]. Let $Y$ and $M$ be topological spaces; then

$$
\begin{aligned}
Y \tau M \Longleftrightarrow & \text { for arbitrary closed subset } A \subset Y \text { and arbitrary } \\
& \text { continuous map } \varphi: A \rightarrow M \text { there exists a continuous } \\
& \text { extension } \bar{\varphi}: Y \rightarrow M .
\end{aligned}
$$

We will consider only the case when $M$ is a $C W$-complex.

Proposition 1. Let $A$ be a closed subset of $Y$. Then the property $Y \tau M$ implies the property $A \tau M$.

Proposition 2. Let $U$ be an open subset of a metric space $Y$. Then $Y \tau M$ implies $U \tau M$.

Proof. There exists a filtration $F_{1} \subset F_{2} \subset \cdots \subset F_{i} \subset \cdots$ of $U$, where $F_{i}=$ $Y-O_{1 / i}(Y-U)$, where $O_{\varepsilon}(B)$ denotes the open $\varepsilon$-neighborhood of $B$ in $Y$. For arbitrary $\varphi: A \rightarrow M$ by induction construct a sequence of maps $\varphi_{i}: A \cup$ $F_{i} \rightarrow M$ with the property $\left.\varphi_{i+1}\right|_{A \cup F_{i}}=\varphi_{i}$ and $\left.\varphi_{i}\right|_{A}=\varphi$ for all $i$. The union $\bigcup \varphi_{i}$ is a continuous extension of the map $\varphi$ to $U$.

Proposition 3. Let $B$ be a closed subset in $Y$. Then the properties $B \tau M$ and $(Y-B) \tau M$ imply $Y \tau M$.

Proof. Suppose that $\varphi: A \rightarrow M$ is an arbitrary map and $A$ is an arbitrary closed subset in $Y$. Due to the property $B \tau M$ there exists an extension $\varphi^{\prime}: A \cup$ $B \rightarrow M$. Since $M \in A N E$ there exists an extension $\bar{\varphi}: O \rightarrow M$ to an open set $O \supset A \cup B$. Let $W$ be an open set with $A \cup B \subset W \subset[W] \subset O$ where $[W]$ is the closure of $W$. Apply $Y-B \tau M$ to extend the map $\left.\bar{\varphi}\right|_{\partial W}$ to the map $\widetilde{\varphi}: Y-W \rightarrow M$. The union $\left.\tilde{\varphi} \cup \bar{\varphi}\right|_{[W]}$ is a continuous map $\psi: Y \rightarrow M$ with the restriction $\left.\psi\right|_{A}=\varphi$.

Proposition 4. Suppose that compact $Y$ is a union $\bigcup_{i=1}^{m} F_{i}$ of closed subsets. Then the properties $F_{i} \tau M$ imply $Y \tau M$.

Proof. By induction on $m$ and using Proposition 1.

Let $\Sigma M$ denote the suspension of $M$.

Lemma 1. The property $X \tau M$ implies $(X \times[0,1]) \tau \Sigma M$ for metric spaces $X$. Proof. Define an open set $V(r, U)=\{(x, t) \in X \times[0,1] \mid x \in U\}$ and $r-$ $d(x, X-U)<t<r+d(x, X-U)$ where $U$ is an open subset in $X$ and $d$ is the metric on $X$. Then for every $V(r, U)$ the boundary $\partial V(r, U)$ in $X \times R$ is equal to the union $F_{1} \cup F_{2}$ where

$$
F_{1}=\left\{\left(x, t_{1}: t=\max \{0, r-d(x, X-U)\}\right.\right.
$$

and

$$
F_{2}=\{(x, t): t=\min \{1, r+d(x, X-U)\} .
$$


It is easy to see that $F_{i}, i=1,2$ is homeomorphic to the closure [U] of $U$. since $F_{i} \tau M$ by Proposition 4 we have $\partial V \tau M$.

Let $A \subset X \times[0,1]$ be a closed subset and $\varphi: A \rightarrow \Sigma M$ be a continuous map. The suspension $\Sigma M$ consists of the union of two cones: $\Sigma M=$ $\operatorname{con}^{+} M \cup \operatorname{con}^{-} M$ with the vertices $x^{+}$and $x^{-}$. Denote $\varphi^{-1}\left(x^{+}\right)=A^{+}$and $\varphi^{-1}\left(x^{-}\right)=A^{-}$. Since the sets $V(r, U)$ generate a basis of the topology on $X \times[0,1]$ there exists an open set $V$ such that $A^{+} \subset V, A^{-} \subset X \times[0,1]-[V]$ and $V=\bigcup_{i=1}^{m} V\left(r_{i}, u_{i}\right)$. Since $\partial V \subset \bigcup_{i=1}^{m} \partial V\left(r_{i}, u_{i}\right)$, Proposition 1 and 4 imply that $\partial V \tau M$. Since $\Sigma M-\left\{x^{+}, x^{-}\right\}$is homeomorphic to $M \times \mathbb{R}$ there exists an extension $\varphi^{\prime}: \partial V \rightarrow \Sigma M-\left\{x^{+}, x^{-}\right\}$of the map $\left.\varphi\right|_{A \cap \partial V}$. Let $\psi$ be the union of $\varphi^{\prime}$ and $\varphi$. Consider the restrictions $\psi^{+}=\left.\psi\right|_{(A \cap[V]) \cup \partial V}$ and $\psi^{-}=\left.\psi\right|_{(A-V) \cup \partial V}$. Since spaces $\operatorname{con}^{+} M$ and $\operatorname{con}^{-} M$ are contractable there are extensions $\bar{\psi}^{+}:[V] \rightarrow \operatorname{con}^{+} M$ and $\bar{\psi}^{-}: X \times[0,1]-V \rightarrow \operatorname{con}^{-} M$ of $\psi^{+}$ and $\psi^{-}$. The union $\bar{\psi}^{+} \cup \bar{\psi}^{-}$gives the map $\bar{\psi}: X \times[0,1] \rightarrow \Sigma M$ which is an extension of $\varphi$.

Proposition 5. The property $X \tau M$ implies con $X \tau \Sigma M$.

Proof. By virtue of Lemma 1, $X \times[0,1] \tau \Sigma M$. By Proposition 2 we have $X \times[0,1) \tau \Sigma M$. Since $p t \tau \Sigma M$ and $\operatorname{con} X-p t \approx X \times[0,1)$ Proposition 3 implies con $X \tau \Sigma M$.

The following proposition has a similar proof.

Proposition 6. The property $X \tau M$ implies $\Sigma X \tau \Sigma M$.

Corollary. $\forall i X \tau M$ implies $\Sigma^{i} X \tau \Sigma^{i} M$.

Theorem 3. For arbitrary $C W$-complex $M$ and compactum $X$ the property $X \tau M$ implies the property $X \tau \Omega^{i} \Sigma^{i} M$ for any $i=1,2, \ldots, \infty$.

Here $\Omega^{i} Y$ denotes iterated loop space.

Proof. Let $i<\infty$. Consider the diagram

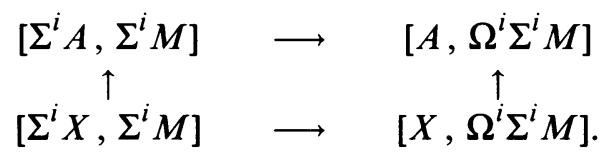

All horizontal arrows are isomorphisms, the left vertical arrow is an epimorphism due to the corollary of Proposition 6. Thus the right vertical arrow is an epimorphism too. Hence $X \tau \Omega^{i} \Sigma^{i} M$.

Recall that $\Omega^{\infty} \Sigma^{\infty} M=\underline{\lim } \Omega^{i} \Sigma^{i} M$. Then compactness of $X$ and the properties $X \tau \Omega^{i} \Sigma^{i} M$ for $i<\infty$ imply the property $X \tau \Omega^{\infty} \Sigma^{\infty} M$.

Remark 2. $X \tau \Omega^{\infty} \Sigma^{\infty} S^{n} \Longleftrightarrow \operatorname{dim}_{S} X \leq n$ where $\operatorname{dim}_{S}$ is the stable cohomotopy dimension [D3]. So, for $M=S^{n}$, Theorem 3 claims the inequality $\operatorname{dim} X \geq \operatorname{dim}_{S} X$. 
Problem. Does $X \tau M$ imply $X \tau S P^{\infty} M$, where $S P^{\infty}$ is the infinite symmetric power?

\section{SPANIER-WhiteheAd DUALITY}

Lemma 2. Let $U$ be an open $n$-dimensional ball and $X \subset U$ be a closed subset. Let $M=U-X$, and let $X^{\prime}$ be the one-point compactification of $X$. Then for a finite-dimensional compactum $Y$ and for large enough $m$ there is an isomorphism $\beta_{Y}:\left[Y, \Omega^{m} \Sigma^{m} M\right] \rightarrow\left[\Sigma^{m}\left(Y \wedge X^{\prime}\right), S^{m+n-1}\right]$ which depends naturally on $Y$.

A proof of Lemma 2 actually is contained in [D4] (see the lemma) and it is a consequence of Spanier-Whitehead duality.

Consider the one-point compactification $U^{\prime}$ of $U$. In the $n$-dimensional sphere $U^{\prime} \simeq S^{n}$ choose a decreasing sequence $\left\{K_{i}\right\}$ of polyhedra with intersection $\bigcap K_{i}=X^{\prime}$. Spanier-Whitehead duality claims that for finite-dimensional $Y$ and large enough $m$ there is an isomorphism $\beta_{i}:\left[\Sigma^{m} Y, \Sigma^{m}\left(U^{\prime}-K_{i}\right)\right] \rightarrow$ $\left[\Sigma^{m}\left(Y \wedge K_{i}\right), S^{m+n-1}\right][\mathrm{Sp}]$. The limit $\lim _{i \rightarrow \infty} \beta_{i}$ gives the isomorphism $\beta_{Y}$. This conclusion is based on the following propositions.

Proposition 7. Let the compactum $X$ be a limit space of an inverse system $\left\{X_{i}, p_{i}^{i+1}\right\}$ of compacta and let $M$ be a CW-complex. Then $[X, M]=$ $\stackrel{\lim }{\longrightarrow}\left[X_{i}, M\right]$.

Proposition 8. Suppose $M$ is a limit space of a direct system $\left\{M_{i}, \varphi_{i}^{i+1}\right\}$ of $C W$-complexes and inclusions. Then for any compactum $Y$ there is an equality $[Y, M]=\lim _{\longrightarrow}\left[Y, M_{i}\right]$.

Lemma 3. Let $X$ be a compact subset of $\mathbb{R}^{n}$, then for compactum $Y$ the following are equivalent:

(1) the space $C\left(Y, \mathbb{R}^{n}-X\right)$ is dense in $C\left(Y, \mathbb{R}^{n}\right)$,

(2) for any open ball $U \subset \mathbb{R}^{n}, Y \tau M_{U}$, where $M_{U}=U-X$.

Proof. (1) $\Rightarrow$ (2). Let $A$ be a closed subset of $Y$ and $\varphi: A \rightarrow M_{U}$ be an arbitrary map. Choose an arbitrary extension $\varphi^{\prime}: Y \rightarrow U$. There exists $\varepsilon>0$ such that every map $\psi \varepsilon$-close to $\varphi$ is homotopic to $\psi$ in $M_{U}$. Approximate $\varphi^{\prime}$ by $\psi: Y \rightarrow \mathbb{R}^{n}-X$ such that $\psi(Y) \subset M_{U}$ and $\psi$ is $\varepsilon$-close to $\varphi^{\prime}$. The homotopy extension theorem implies that there is an extension $\dot{\bar{\varphi}}: Y \rightarrow M_{U}$ of $\varphi$.

(2) $\Rightarrow$ (1) See the proof of Theorem 1 in [D1].

\section{Proof of Theorem 1}

Suppose the contrary: $\operatorname{dim} X \times Y \geq n$. We can assume that $\operatorname{dim} X \times Y=n$. (Otherwise consider $X=X \times\{0\} \subset \mathbb{R}^{n} \times \mathbb{R}^{m-n}=\mathbb{R}^{m}$ where $m=\operatorname{dim} X \times Y$. It is easy to see that $C\left(Y, \mathbb{R}^{m}-X\right)$ is dense in $C\left(Y, \mathbb{R}^{m}\right)$. The case of $\operatorname{dim} Y=$ 
$\infty$ is excluded because if $\operatorname{dim} Y \geq n$ then always there is an essential map of $Y$ onto $n$-dimensional cube.)

Let $Z$ be a cone over $Y$. Lemma 3 implies the property $Y \tau M_{U}$ for an arbitrary open ball $U \subset \mathbb{R}^{n}$. By virtue of Proposition 5 we have $Z \tau \Sigma M_{U}$ for every open ball $U \subset \mathbb{R}^{n}$. Denote $W=U \cap X$ and let $W^{\prime}$ be the onepoint compactification of $W$. Let $V$ be an arbitrary open subset of $Z$ and $A=Z-V$.

The set $U$ is naturally embedded in $\Sigma U$ as the equator. Then $\Sigma U-W$ is homotopy equivalent to $\Sigma M_{U}$. By Lemma 2 there is the following diagram

$$
\begin{aligned}
& {\left[A, \Omega^{k} \Sigma^{k} \Sigma M_{U}\right] \stackrel{\beta_{1}}{\longrightarrow}\left[W^{\prime} \wedge A, \Omega^{k} \Sigma^{k} S^{n}\right]} \\
& \uparrow \alpha_{1} \quad \uparrow \alpha_{2} \\
& {\left[Z, \Omega^{k} \Sigma^{k} \Sigma M_{U}\right] \stackrel{\gamma_{1}}{\longrightarrow}\left[W^{\prime} \wedge Z, \Omega^{k} \Sigma^{k} S^{n}\right],}
\end{aligned}
$$

where for large enough $k$ all horizontal arrows are isomorphisms. Theorem 3 implies that $\alpha_{1}$ is an epimorphism. Therefore $\alpha_{2}$ is an epimorphism.

Since $\operatorname{dim}\left(Z \wedge W^{\prime}\right)=n+1<2 n-1$ the homomorphisms $\beta_{2}, \gamma_{2}$ in the following diagram are isomorphisms:

$$
\begin{array}{ccc}
{\left[W^{\prime} \wedge A, \Omega^{k} \Sigma^{k} S^{n}\right]} & \stackrel{\beta_{2}}{\longleftarrow} & {\left[W^{\prime} \wedge A, S^{n}\right]} \\
\uparrow \alpha_{2} & & \uparrow \alpha_{3} \\
{\left[W^{\prime} \wedge Z, \Omega^{k} \Sigma^{k} S^{n}\right]} & \stackrel{\gamma_{2}}{\longleftarrow} & {\left[W^{\prime} \wedge Z, S^{n}\right] .}
\end{array}
$$

Hence $\alpha_{3}$ is an epimorphism.

Since $\operatorname{dim} W^{\prime} \times Z=n+1$ then for homomorphism $\beta_{3}, \gamma_{3}$ in the diagram

$$
\begin{array}{ccc}
{\left[W^{\prime} \wedge A, S^{n}\right]} & \stackrel{\beta_{3}}{\rightarrow} & {\left[W^{\prime} \wedge A, K(\mathbb{Z}, n)\right]} \\
\uparrow \alpha_{3} & & \uparrow \alpha_{4} \\
{\left[W^{\prime} \wedge Z, S^{n}\right]} & \stackrel{\gamma_{3}}{\longrightarrow} & {\left[W^{\prime} \wedge Z, K(\mathbb{Z}, n)\right],}
\end{array}
$$

are epimorphisms. Therefore $\alpha_{4}$ is an epimorphism. Here $K(\mathbb{Z}, n)$ is the Eilenberg-MacLane complex.

Consider the cohomology exact sequence of the pair $\left(W^{\prime} \wedge Z, W^{\prime} \wedge A\right): \cdots \leftarrow$ $\check{H}^{n+1}\left(W^{\prime} \wedge Z\right) \leftarrow \check{H}^{n+1}\left(W^{\prime} \wedge Z, W^{\prime} \wedge A\right) \leftarrow \check{H}^{n}\left(W^{\prime} \wedge A\right) \stackrel{\alpha_{4}}{\leftarrow} \check{H}^{n}\left(W^{\prime} \wedge Z\right)$. Since $Z$ is contractible then $\breve{H}^{*}\left(W^{\prime} \wedge Z\right)=0$. This and the fact that $\alpha_{4}$ is an epimorphism imply that $\check{H}^{n+1}\left(W^{\prime} \wedge Z, W^{\prime} \wedge A\right)=0$. Because $\left(W^{\prime} \wedge Z\right) /\left(W^{\prime} \wedge A\right)=\left(W^{\prime} \times Z\right) /\left(W^{\prime} \times A\right) \cup(*) \times Z$ (where $\left.(*)=W^{\prime}-W\right)$ then $\check{H}^{n+1}\left(W^{\prime} \times Z, W_{U}^{\prime} \times A \cup(*) \times Z\right)=0$. In other terms $H_{c}^{n+1}(W \times V)=0$. Since the sets of the form $W \times V$ are a basis in $X \times Z$ we have [Kuz] the inequality $c-\operatorname{dim}_{\mathbb{Z}}(X \times Z)<n+1$. Since both $X$ and $Y$ are finite-dimensional then $\operatorname{dim}(X \times \operatorname{con} Y)<n+1$. We have reached a contradiction.

\section{REFERENCES}

[D1] A. N. Dranishnikov, An intersection of compacta in Euclidean space, Proc. Amer. Math. Soc. (to appear). Homological dimension theory, Uspekhi Mat. Nauk 434 (1988), 11-55. (Russian) 
[D3] _ Generalized cohomological dimension of compact metric spaces, Tsukuba Math. J. 14 (1990).

[D4] _ Spanier-Whitehead duality and stability of intersections of compacta, Trudy Mat. Inst. Steklov. (to appear). (Russian)

[Ku] K. Kuratowski, Topology, vol. 2, Academic Press, New York and London, 1968.

[Kuz] V. I. Kuzminov, Homological dimension theory, Uspekhi Mat. Nauk 235 (1968), 3-49; Russian Math. Surveys 235 (1968), 1-45. (Russian)

[Sp] E. H. Spanier, Function spaces and duality, Ann. of Math. 70 (1959), 338-378.

Department of Mathematics, Cornell University, Ithaca, New York 14853

Current address: Steklov Institute of Mathematics, Ulitsa Vavilov 42, 117966 Moscow, USSR 